\title{
Femtosecond electron dynamics of image-potential states on clean and oxygen-covered $\operatorname{Pt}(111)$
}

\author{
S. Link, H. A. Dürr, G. Bihlmayer, S. Blügel, and W. Eberhardt \\ Institut für Festkörperforschung, Forschungszentrum Jülich GmbH, D-52425 Jülich, Germany \\ E. V. Chulkov, ${ }^{1,2}$ V. M. Silkin, ${ }^{1}$ and P. M. Echenique ${ }^{1,2,3}$ \\ ${ }^{1}$ Donostia International Physics Center (DIPC), 20018 San Sebastián, Basque Country, Spain \\ ${ }^{2}$ Departamento de Física de Materiales, Facultad de Ciencias Químicas, Universidad del Pais Basco/Euskal Herriko Unibertsitatea, \\ Apartado 1072, 20018 San Sebastián/Donostia, Spain \\ ${ }^{3}$ Centro Mixto CSIC-UPV/EHU 20018 San Sebastián, Basque Country, Spain
}

(Received 28 September 2000; published 2 March 2001)

\begin{abstract}
We have investigated the lifetimes of image-potential states on the $\operatorname{Pt}(111)$ surface with time-resolved two-photon photoemission and first-principles calculations. Electrons populating the first two image-potential states decay into bulk states after $26 \pm 7$ and $62 \pm 7 \mathrm{fs}$, respectively. This is in agreement with results of theoretical calculations. Oxygen adsorption reduces the image-potential state lifetimes by a factor of 2 . This is caused by a strong change of the electronic structure near the Fermi level.
\end{abstract}

DOI: 10.1103/PhysRevB.63.115420 PACS number(s): 73.20. $-\mathrm{r}$, 78.47.+p, 73.20.At, 79.60.-i

\section{INTRODUCTION}

The reduced dimensionality at a solid surface can introduce additional electronic states localized at the solidvacuum interface. These surface states are of interest in epitaxy since they were found to modify energy barriers for adatom diffusion. ${ }^{1}$ A special class of surface states are the so called image-potential states (IPSs). They occur if an electron is trapped in front of a metal surface by its own image charge. If a gap in the bulk electronic density of states (DOS) in the solid prevents the electron from decaying into the crystal a Rydberg series of bound IPSs can form. Theoretical analysis showed ${ }^{2}$ that these states occur at binding energies $E_{B}=e_{v}^{0}-0.85 /(n+a)^{2} e V$ converging towards the vacuum level $e_{0}^{v}$. Here, $n$ is the IPS quantum number and $a$ is a quantum defect with values $0<a \leqslant 0.5$ related to the phase change upon electron reflection at the crystal surface. ${ }^{3}$ Electrons in the normally unoccupied IPSs are localized in front of the crystal and experience the full translational symmetry for motion parallel to the surface. Thus, IPSs can provide model systems to study excitations of a two-dimensional electron gas.

Experimental detection of IPSs involves populating these states with electrons. This has been done either by adding an additional electron to the solid as in inverse photoemission spectroscopy (IPE) ${ }^{4}$ or by photoexciting electrons from occupied states followed by a second photoemission step. This two-photon photoemission spectroscopy (2PPE) has become increasingly popular with the development of suitable highpower laser light sources. ${ }^{5}$ Electron lifetimes of IPSs have initially been determined from the intrinsic linewidths of these states probed with both IPE and 2PPE. A more recent development is the utilization of spin polarization in IPE (Ref. 6) and variable light polarization in $2 \mathrm{PPE}^{7}$ Such measurements allowed the determination of magnetic exchange splitting of IPSs (Refs. 6,7) and the observation of different relaxation times of spin-up and -down electrons ${ }^{6,8}$ at the surfaces of ferromagnets.

A breakthrough in the accurate measurement of IPS life- times has been time-resolved 2PPE (TR2PPE) utilizing advances in the generation of femtosecond laser pulses. ${ }^{9}$ In TR2PPE a pump pulse is used to populate the IPEs. The evolution of the IPS population is then probed in real time with a time-delayed second laser pulse. Measurements on noble metal surfaces have resulted in a wealth of data on the decay of IPSs (Refs. 10,11) that can be understood quantitatively within a many-particle framework of energy relaxation processes at surfaces. ${ }^{12,13}$ The lifetime of IPSs is determined by different factors. On noble metal (100) surfaces the IPSs are located near the center of a gap in the electronic DOS. They decay mainly through electron-hole pair creation and a lifetime increase proportional to $n^{3}$ is expected in good agreement with experiments. ${ }^{10}$ The situation is more complex at the close-packed noble metal (111) surfaces. In this case the IPSs are located near the upper edge of a gap in the bulk electronic DOS. With increasing quantum number, $n$, the IPSs become energetically degenerate with bulk states and can decay into them. As a consequence the lifetime is observed to be reduced with increasing $n .^{11}$ The lifetime of the $n=1$ IPS was found to be strongly influenced by other surface states closer to the Fermi level. ${ }^{14}$

In contrast to noble metals the decay of IPSs on transition metal surfaces is more poorly understood. Early linewidth measurements on $\mathrm{Ni}(111)$ indicated a very short lifetime of the $n=1$ IPS of less than $10 \mathrm{fs}{ }^{15}$ This is most likely a consequence of the large $3 d$ DOS bisecting the Fermi level which provides a very effective decay channel due to electron-hole pair creation. ${ }^{15,8}$ A significant lifetime increase for the $n=1$ IPS has been observed on $\operatorname{Pd}(111)$ (Ref. 16) where the $4 d$ band occupation is higher compared to that of the Ni $3 d$ states. The experimental value of $24 \mathrm{fs}$ is in good agreement with theory. ${ }^{16}$ Unfortunately, no lifetimes for higher $n$ IPS could be measured with TR2PPE on Ni(111) and $\operatorname{Pd}(111)$ due to technical restrictions of the available photon energy. In this paper we used TR2PPE and firstprinciples calculations to investigate the lifetimes of the $n$ $=1$ and $n=2$ IPSs on Pt(111). We find a similar lifetime of the $n=1$ IPS compared to $\operatorname{Pd}(111)$ in excellent agreement 
with theory. In contrast to noble metal (111) surfaces we find a longer lifetime for the $n=2$ IPS. Upon oxygen adsorption we find a significant decrease of both IPSs lifetimes. This can be accounted for by the changing electronic DOS near the Fermi level on the $\mathrm{Pt}(111)-(2 \times 2) \mathrm{O}$ surface.

The paper is organized as follows. In the next two sections we describe the experimental setup and theoretical approach, respectively. In Sec. IV the results obtained for the $\mathrm{Pt}(111)$ and $\mathrm{Pt}(111)-(2 \times 2) \mathrm{O}$ surfaces will be presented. This is followed by the discussion of the results in Sec. V. A summary section concludes the paper.

\section{EXPERIMENT}

Ultrashort laser pulses are generated by a Ti:sapphire laser system pumped by an Ar-ion laser (Coherent Innova $400)$. The tunable $(780-860 \mathrm{~nm}$ ) output of an oscillator (Coherent Mira Seed) is first stretched temporally and then coupled into a regenerative amplifier (Coherent RegA). Subsequent temporal compression results in pulses with a total energy of up to $5 \mu \mathrm{J}$, a bandwidth of $25 \mathrm{~nm}$ and a duration of $60 \mathrm{fs}$ at $250 \mathrm{kHz}$ repetition rate. The pulses are frequency doubled using a $\beta$-BBO crystal. This second harmonics output is separated from the fundamental by a dichroic mirror and split into two parts. One part is again frequency doubled. This fourth harmonics serves as the pump pulse to populate the IPSs. The other part of the second harmonics is delayed through traversing an optical delay line and is used to probe the population dynamics of IPSs.

TR2PPE experiments were performed in a UHV chamber at a base pressure in the lower $10^{-10} \mathrm{mbar}$ range. The sample was cleaned by cycles of $\mathrm{Ar}^{+}$sputtering and subsequent annealing to $1000 \mathrm{~K}$. Residual carbon contamination was removed by oxygen adsorption and flashing the sample to 670 $\mathrm{K}$. This procedure resulted in a clean and well ordered $\mathrm{Pt}(111)$ surface as was judged by $2 \mathrm{PPE}$ and low-energy electron diffraction (LEED). Photoelectrons were detected with a hemispherical energy analyzer (Leybold EA200) with 100 meV energy resolution. A bias of $-10 \mathrm{~V}$ was applied to the sample during the 2PPE measurements in order to improve the transmission of the electron spectrometer. We obtained a sample work function of $5.97 \pm 0.03 \mathrm{eV}$ for the clean $\mathrm{Pt}(111)$ surface from $2 \mathrm{PPE}$ experiments in good agreement with results reported previously. ${ }^{17,18}$ Oxygen adsorption at room temperature resulted in a well ordered $p(2 \times 2)$ LEED pattern at a saturation coverage of 0.25 monolayers. ${ }^{19,20}$ The work function for the $\mathrm{Pt}(111)-p(2 \times 2)-\mathrm{O}$ was determined to $6.16 \pm 0.03 \mathrm{eV} .^{19}$

In the 2PPE experiments space charge effects were avoided by using low light fluences and by keeping the photon energies below the work function of the respective surface. The pump and probe photon energies were 5.84 (6.06) and 2.92 (3.03) eV, respectively, for the clean (oxygen saturated) Pt surface. The probe-pulse duration at the sample was $60 \mathrm{fs}$ for both photon energies as was determined from autocorrelation measurements $3 \mathrm{eV}$ above $\mathrm{E}_{F}$ where the estimated lifetimes are below 5 fs. The pump-pulse duration at $5.84 \mathrm{eV}$ photon energy was 140 fs obtained from the crosscorrelation also measured at an energy of $3 \mathrm{eV}$ above $\mathrm{E}_{F}$. At

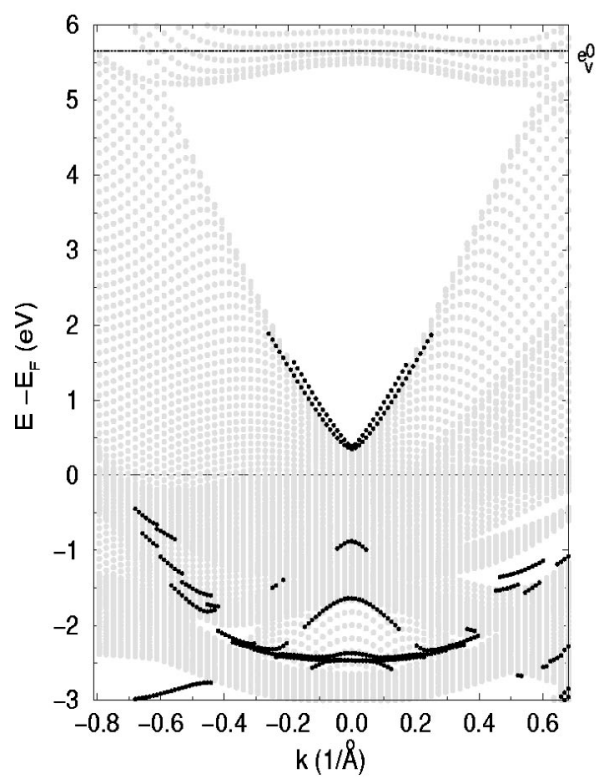

FIG. 1. Projected bulk bandstructure (grey symbols) and states localized at the surface (black symbols) along the $\bar{K}-\bar{\Gamma}-\bar{M}$ direction. $E_{F}$ denotes the Fermi level, $e_{v}^{o}$ the vacuum level of the film calculation. The $\Gamma$ point corresponds to $k=0$. From the $\operatorname{Pt}(111)$ film calculation we plot only the states up to $e_{v}^{o}$ that had either more than $50 \%$ of their charge localized in the surface layer or more than $10 \%$ in the vacuum. Surface states appear in the gaps of the projected bulk band structure.

a photon energy of $6.06 \mathrm{eV}$ the pump-pulse duration was 260 fs.

\section{THEORY}

For better understanding of the electron dynamics in IPS on the clean $\operatorname{Pt}(111)$ surface we have initially calculated the surface electronic structure with the use of the ab initio fullpotential augmented plane wave (FLAPW) method. ${ }^{21}$ The generalized gradient approximation ${ }^{22}$ of the density functional theory was employed to describe the exchangecorrelation potential. Spin-orbit coupling was included in a pseudopertubative manner. ${ }^{23}$ The surface was modeled by a 13 layer film embedded in a semi-infinite vacuum. This thickness is necessary to prevent interaction between the two surfaces of the film, although a small splitting of the $s p$-derived surface state at $0.4 \mathrm{eV}$ above the Fermi level at the Brillouin-zone center $\bar{\Gamma}$ resulting from this interaction can still be observed. Figure 1 shows the $\operatorname{Pt}(111)$ projected bulk bandstructure (gray symbols) and states localized at the surface (black symbols). The unoccupied $s p$-like surface state is visible in early IPE experiments about $0.5 \mathrm{eV}$ above $E_{F} .{ }^{24}$ An occupied $d$-derived surface resonance observed below $E_{F}$ in Fig. 1 has been reported in photoemission experiments. ${ }^{1,17}$

The information obtained from the first-principles calculations was used to construct a one-dimensional model potential along the lines of Ref. 25. To this end we used the width and position of the energy gap at $\bar{\Gamma}$, the $s p$-surface state energy position of $0.4 \mathrm{eV}$ together with the experimen- 
tally determined binding energy of $-0.65 \mathrm{eV}$ relative to $e_{v}^{0}$ for the $n=1$ image state. From this potential one-electron wave functions

$$
\psi_{\kappa \overline{\mathbf{k}}}(\mathbf{r})=\frac{1}{L} e^{i \overline{\mathbf{k r}}} \cdot \varphi_{\kappa}(z)
$$

where $L$ is a normalization length, and one-electron energies

$$
E_{\kappa \overline{\mathbf{k}}}=E_{\kappa}+\frac{\overline{\mathbf{k}}^{2}}{2 m}
$$

were evaluated in order to calculate the damping rate (inverse lifetime) of the $n=1,2$ IPSs. In Eqs. (1) and (2) $\kappa$ numerates electron bands and $\overline{\mathbf{k}}$ is the two-dimensional electron wave vector. We computed the inverse lifetime within a many-body theory where the decay rate of an excited electron $\left(E>E_{F}\right)$ in a quantum state $\psi(\mathbf{r})$ with energy $E$ was obtained as the projection of the imaginary part of the selfenergy $\Sigma(\mathbf{r}, \mathbf{r} ; E)$ onto the state itself. The self-energy was calculated within the $G W$ approximation ${ }^{26}$ when only the first term in the series expansion of $\Sigma$ in terms of the screened Coulomb interaction $W$ is used. We also replaced the full one-electron Green function $G$ by the noninteracting Green function $G_{0}$. The technique of the calculation of the decay rate of image states was described in detail in Refs. $12,16,13$ where it was used for evaluations of the lifetime of IPSs on $\mathrm{Cu}(100), \mathrm{Cu}(111)$, and $\mathrm{Pd}(111)$ surfaces. We do not give more details of the calculation here and refer readers to Refs. $12,16,13$. Though our model does not contain $d$ electrons it is reasonably accurate for description of electron dynamics in image states on $\mathrm{Cu}(100)$ and $\mathrm{Cu}(111)$. In bulk $\mathrm{Cu}$ the $3 d$ states are totally occupied and lie in an energy interval of $2-5 \mathrm{eV}$ below $E_{F}$ and transitions involving these states are not expected to play a direct role. On the other hand bulk Pd and Pt possess empty $d$ orbitals which lie in a narrow energy interval of 0.3 just above $E_{F}$. However, the total number of unoccupied $d$ states is small and they are not expected to give a dominant contribution to the decay rate of IPSs. This is confirmed on $\operatorname{Pd}(111)$ where the theoretical model gives results which are in good agreement with TR2PPE measurements. ${ }^{16}$ Our recent first-principles calculations ${ }^{27}$ of the decay rate of the surface $(n=0)$ and image $(n=1)$ states on $\operatorname{Pd}(111)$ confirm this conclusion. For the 50 layer $\mathrm{Pt}(111)$ film we obtained lifetimes of $\tau_{1}=29 \mathrm{fs}$ and $\tau_{2}=73$ fs for the $n=1$ and $n=2$ IPSs, respectively.

\section{RESULTS}

\section{A. The clean $\operatorname{Pt}(111)$ surface}

Energy resolved 2PPE spectra of IPSs on the clean $\mathrm{Pt}(111)$ surface are shown in Fig. 2. The spectra were taken at different time delays between pump and probe pulses. The first two states with quantum numbers $n=1$ and $n=2$ are clearly resolved with binding energies of $-0.65 \pm 0.05$ and $-0.16 \pm 0.05 \mathrm{eV}$, respectively. The energy position of the first image potential state is close to the value of $0.63 \mathrm{eV}$ obtained from IPS, ${ }^{28}$ but is $0.13 \mathrm{eV}$ smaller than that reported by Kinoshita et al. ${ }^{18}$ With increasing delay time be-

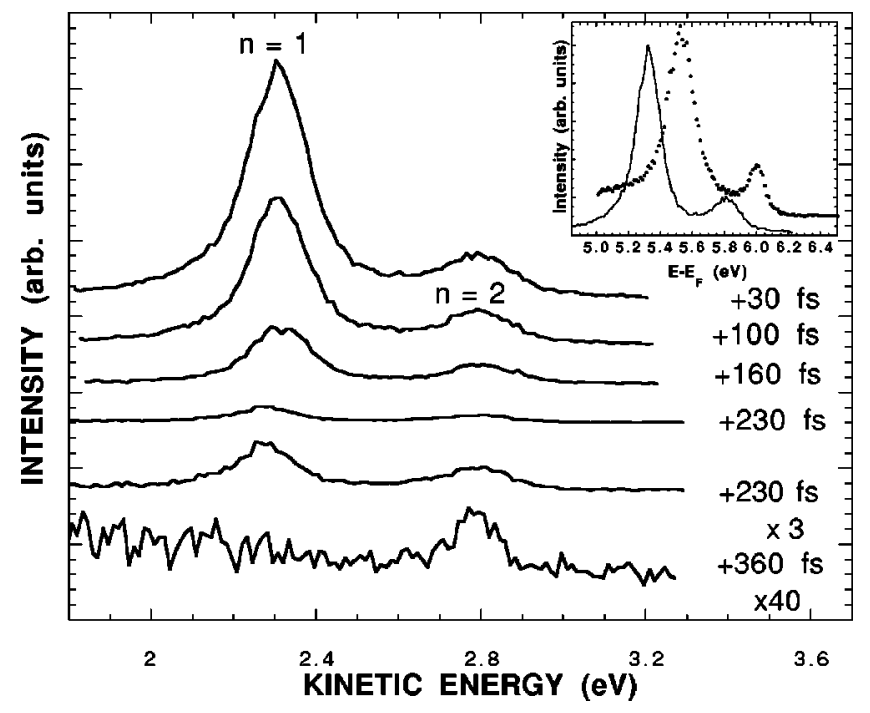

FIG. 2. Energy distribution curves for the two resolved image potential states on the clean $\mathrm{Pt}(111)$ surface at different delay times after excitation. The inset shows the energy shift of the image potential states with respect to $E_{F}$ after oxygen adsorption (dotted line). The image potential states on the clean surface are also shown (solid line).

tween pump and probe pulses the relative intensities between both IPSs change. At a delay time of 340 fs only the $n=2$ IPS is still occupied whereas the first state is completely depopulated. This is clear evidence of a longer lifetime for the $n=2$ state. Another effect seen in the spectra especially for the $n=2$ image-potential state is the decrease in the spectral width with increasing delay time. This broadening at short delay times has been observed earlier on other surfaces ${ }^{10}$ and was attributed to the contribution of a resonant two-photon photoemission process occurring when pump and probe pulses are in temporal overlap near zero time delay. ${ }^{29}$ The intrinsic linewidth of the IPSs has to be determined at sufficient delay between pump and probe pulse, when the pump pulse has already passed by. An analysis of the spectra at sufficient delay times gives the lifetime dominated linewidths of $30 \pm 15$ and $18 \pm 12 \mathrm{meV}$ for the $n$ $=1$ and $n=2$ state, respectively.

We determined the image potential state lifetimes by setting the energy analyzer to the photoelectron kinetic energy values corresponding to the IPS peaks in Fig. 2. Then the delay between pump and probe-pulse was varied. The results are shown in Fig. 3 for the $\operatorname{Pt}(111) n=1$ and $n=2$ IPS. The instrumental cross-correlation function was obtained on a contaminated $\mathrm{Pt}(111)$ surface where all image potential states were quenched. The full width at half maximum of the cross-correlation trace was deduced to 152 fs by fitting a $\operatorname{sech}^{2}$ function to the data (dashed line in Fig. 3). The maximum of the transient response of the $n=1$ IPS is shifted by 14 fs towards positive delay times. In addition the curve is slightly broadened indicating a clearly resolved finite lifetime of this state. ${ }^{11} \mathrm{~A}$ by far stronger effect is seen in the transient response of the $n=2$ state. The curve becomes significantly broader and shows an asymmetric line shape corroborating the longer lifetime seen in the $2 \mathrm{PPE}$ spectra of Fig. 2. 


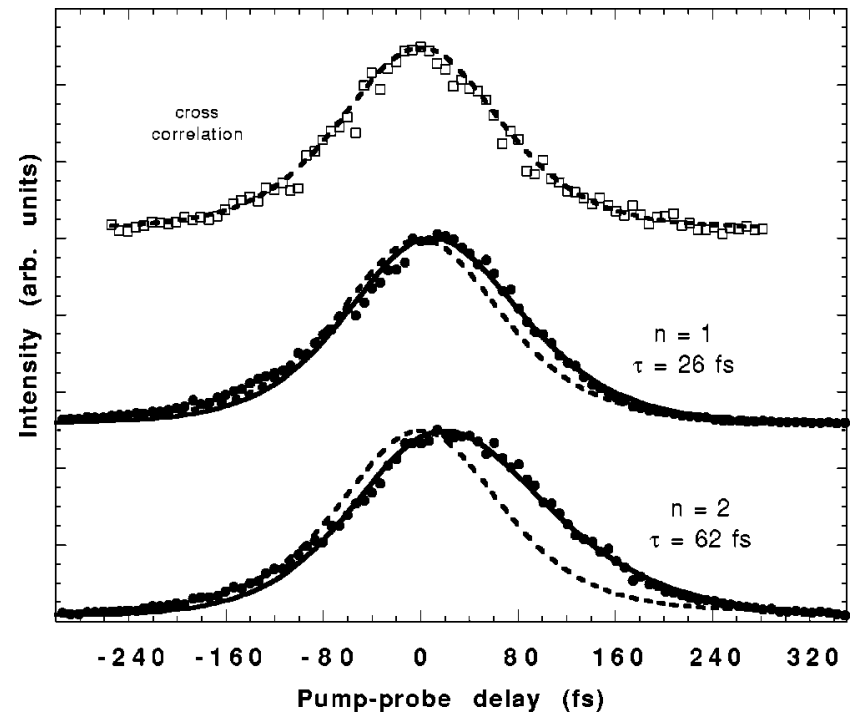

FIG. 3. Transient responses of the $n=1$ (middle panel) and $n$ $=2$ (bottom panel) image potential states (solid symbols). The cross correlation trace (open squares, top panel) reflects the instrumental function described by a $\operatorname{sech}^{2}$ function (dashed lines). From fits to the data lifetimes of $26 \pm 7 \mathrm{fs}$ and $62 \pm 7 \mathrm{fs}$ were obtained for the $n=1$ and $n=2$ state, respectively.

In order to quantitatively evaluate the population of the IPSs one has to take into account the influence of the pumppulse laser field $E(t) \sin (\omega t)$ as long as the excitation takes place. Here $E(t)$ denotes the envelope of the pump pulse and $\omega$ is the frequency of light. This process is described by electronic transitions in a two-level system interacting with a time dependent oscillatory optical perturbation and can be described by using the optical Bloch equations in the rotating wave approximation. ${ }^{30,31}$ A pump pulse of sufficiently narrow bandwidth can generate coherent fluctuations of the IPS population. These oscillations, however, are smeared out in the case of a broad-band excitation when the frequencey spread of the light is larger than the linewidth of the excited final state ${ }^{30}$ In our experiment the bandwidth of the laser pump-pulse can be estimated from the measured bandwidth of the $1.46 \mathrm{eV}$ output $(40 \mathrm{meV})$ of the Ti:sapphire laser system. Assuming that there are no energy cutoffs in the frequency spectrum of the laser pulse after frequency conversion $^{32,33}$ the estimated bandwidth exceeds the measured intrinsic linewidth of the $n=1$ image potential state. In this case the description of the IPS final-state population simplifies from solving the Bloch equations to a rate equation treatment. ${ }^{30}$ Using a sech ${ }^{2}$ function to describe the temporal laser-pulse shape we obtain lifetimes of $\tau_{1}=26 \pm 7 \mathrm{fs}$ and $\tau_{2}=62 \pm 7$ fs for the $n=1$ and $n=2$ IPS on $\operatorname{Pt}(111)$, respectively. The measured value of $\tau_{1}$ is in good agreement with the calculated value of $\tau_{1}=29$ fs while the theoretical lifetime $\tau_{2}=73 \mathrm{fs}$ is slightly higher than the experimental one. This small descrepancy between the calculated and measured with TR2PPE lifetimes of the high order image states $n$ $=2,3, \ldots$, is also obtained for $\mathrm{Cu}(100)$ (Ref. 12) and for $\operatorname{Ag}(100) .{ }^{34}$ Despite this discrepancy the agreement between the experimental and theoretical lifetimes seems to be reasonably good.

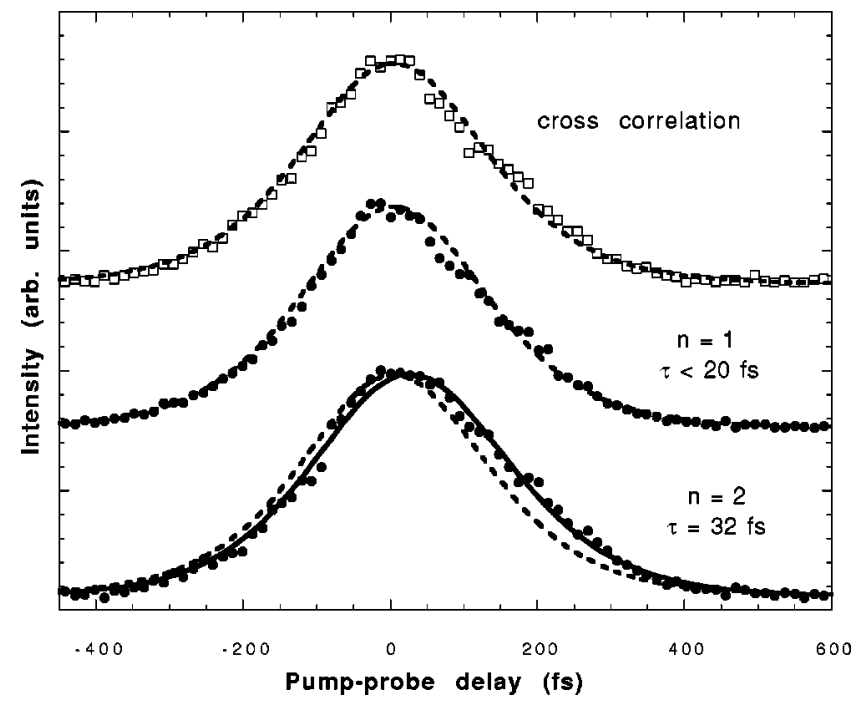

FIG. 4. The transient responses of the image potential states on the oxygen covered surface are shown. It can be seen that the transient response of the first image potential state (middle panel, solid symbols) can be described by the $\operatorname{sech}^{2}$ fit (dashed line) to the instrumental function (open squares). The fit to the $n=2$ state gives a value of $32 \pm 10 \mathrm{fs}$.

\section{B. The $\operatorname{Pt}(111)-p(2 \times 2) O$ surface}

As mentioned above, after oxygen adsorption the work function increases to $6.16 \pm 0.03 \mathrm{eV}$. Since image potential states are pinned to the vacuum level, their relative energy position with respect to $E_{F}$ is changed. The inset of Fig. 2 shows the energy position of the image-potential states relative to $E_{F}$ for the oxygen covered surface (solid circles) in comparison to those of the clean surface (line). We obtained binding energies of $-0.67 \pm 0.05 \mathrm{eV}$ for the $n=1$ and -0.19 $\pm 0.05 \mathrm{eV}$ for the $n=2 \mathrm{IPS}$, respectively. Figure 4 shows the transient responses of the $n=1$ and $n=2$ IPSs on the oxygen covered surface. The instrumental cross correlation with a full width at half maximum of 270 fs is also shown. Unfortunately, this is larger than for the lower photon energy used on the clean Pt(111) surface. However, we could reproduce the IPSs lifetimes on clean $\operatorname{Pt}(111)$ for this higher photon energy. A lifetime of $32 \pm 10$ fs for the $n=2$ state on $\mathrm{Pt}(111)-p(2 \times 2) \mathrm{O}$ was deduced from a fit (solid line) to the data in Fig. 4 (curve C). The lifetime for the $n=1$ IPS is much shorter as can be seen from the transient response which follows directly the instrumental cross-correlation trace. We estimate an upper limit of $20 \mathrm{fs}$ for the lifetime of this state keeping in mind that the temporal resolution is reduced due to the relatively broad cross-correlation trace at this photon energy .

Additional measurements have been performed to investigate the influence of oxygen adsorption on the valence electronic structure. For this purpose we generated laser pulses with a photon energy of $4.47 \mathrm{eV}$ by sum-frequency mixing of the $1.49 \mathrm{eV}$ output of the Ti:sapphire laser system and the frequency-doubled radiation. Figure 5 shows the energy distribution curves of the valence band from the clean (solid line) and oxygen covered (dashed line) Pt(111) surface obtained with 2PPE. A feature at an energy of $0.52 \pm 0.05 \mathrm{eV}$ 


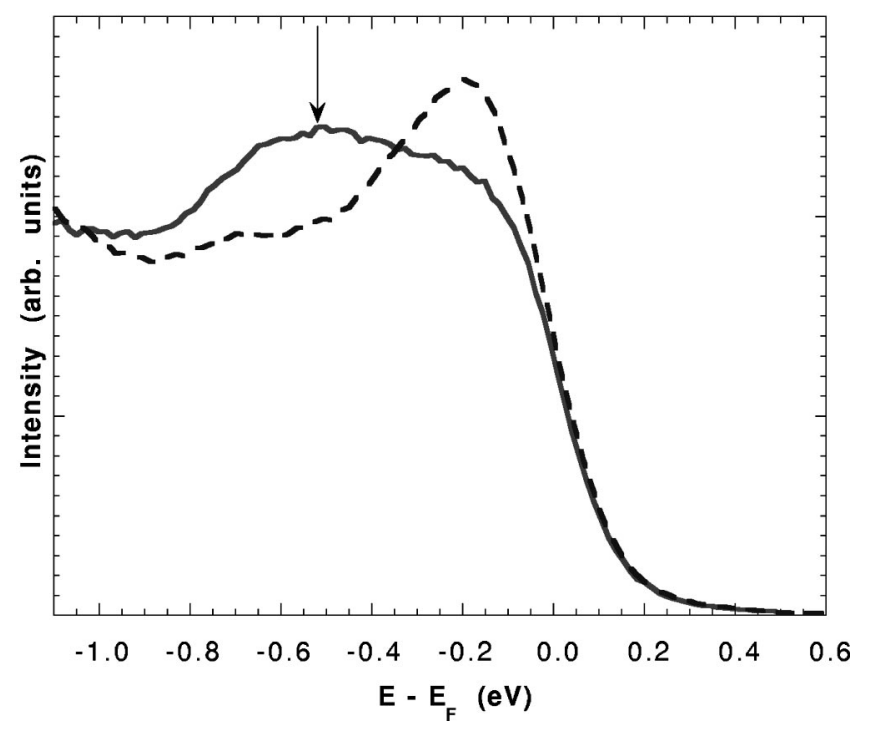

FIG. 5. Energy distribution curves for transitions of the clean (solid line) and oxygen covered (dashed line) occupied states close to $E_{F}$ obtained with a photon energy of $4.47 \mathrm{eV}$. The occupied surface resonance on the clean surface is marked by the arrow.

below $E_{F}$ is seen for the clean surface (marked by an arrow in Fig. 5) which is quenched under oxygen adsorption. This structure was also observed by Roos et al. ${ }^{17}$ who assigned it to an occupied surface resonance. Such a surface resonance is also apparent in the band structure calculations of Fig. 1 near $\bar{\Gamma}$ at energy slightly lower than that observed in the experiments. In addition the DOS near $E_{F}$ is considerably enhanced by oxygen adsorption.

\section{DISCUSSION}

The lifetimes of the image potential states on the clean surface reflect the localization of their wave functions with respect to the metal. The wave function maximum of the $n$ $=2$ state is located further into the vacuum region, thus, resulting in a reduced wave function overlap with energetically adjacent bulk states. A comparison between the experimental data for the binding energies of the image potential states and the calculated band structure seen in Fig. 1 implies that both states are still located inside the bulk band gap. The good agreement for the obtained lifetimes of the image potential states on the clean surface between experiment and theory supports this interpretation since the band structure data from Fig. 1 were taken for the calculation of the image potential state lifetimes. However, the agreement between the measured linewidths and the lifetimes is only satisfying for the first image potential state. The linewidth can be affected by defects of the crystal lattice or dephasing processes. This has also been observed on $\operatorname{Pd}(111)$ for the first image potential state. ${ }^{16}$ The lifetime value for the first image potential state on $\mathrm{Pt}(111)$ of $26 \mathrm{fs}$ is close to the value of 24 fs for the $n=1$ state on $\operatorname{Pd}(111)$. That result might be surprising since on $\operatorname{Pd}(111)$ the vacuum level and the image potential states lie close to the middle of the bulk band gap. For the decay rate $\hbar / \tau$ of the $n=2$ state on $\operatorname{Pd}(111)$ no experimental value is available. But the theoretical value of $7.4 \mathrm{meV}$ (Ref. 16) is close to that of $\mathrm{Pt}(111)$ of $9 \mathrm{meV}$.

The adsorption of oxygen results in a well ordered $\mathrm{Pt}(111)-p(2 \times 2)-\mathrm{O}$ surface structure that was found to involve a slight rearrangement of the $\mathrm{Pt}$ substrate atoms. ${ }^{20}$ Compared to the clean surface, the lifetime of the $n=2$ state is reduced by a factor of 2 , whereas for the lifetime of the first image-potential state only an upper limit can be estimated. Several effects can be responsible for the observed effect.

Schuppler et al. had observed a linewidth broadening of image potential states on a $\operatorname{Ag}(100)$ surface under oxygen adsorption. ${ }^{35}$ The linewidths of the image potential states were observed to increase linearly with oxygen exposure indicating shorter lifetimes because of more effective scattering of electrons in image potential states with $\mathrm{O}$ atoms. This may play a role on a surface with disordered adatoms, but is expected to be negligible on surfaces with well ordered layers of adatoms. ${ }^{36,37}$

Due to the increased work function especially the second image-potential state could be shifted out of the band gap. Therefore its wave function would penetrate deeper into the crystal leading to a stronger coupling with adjacent bulk bands and hence to a shorter lifetime. Such a behavior would mainly affect the $n=2$ image potential state as was observed on $\mathrm{Cu}(111) .{ }^{37}$ However, on the $\mathrm{Pt}(111)-(2 \times 2) \mathrm{O}$ surface this effect is unlikely since all image potential state lifetimes are reduced in a similar way.

For the decay of image potential state electrons into electron-hole pairs other surface states play a dominant role. Since image potential states are strongly localized in the vacuum region, they couple mainly to other surface states while the coupling to bulk bands is relatively smaller. For example, the unoccupied $s-p$ band derived $S_{1}$ surface state is therefore assumed to be one reason for the relatively broad linewidth of the $n=1$ state on $\mathrm{Ni}(111) .{ }^{15}$ As mentioned above, under oxygen adsorption the surface resonance observed $0.52 \mathrm{eV}$ below $E_{F}$ is quenched. It is presently not clear whether this state is simply depopulated and shifted above $E_{F}$ (Ref. 1) or whether a major reorganizazion of the valence DOS occurs. The latter seems more likely since a small oxygen indiced buckling reconstruction of the Pt substrate was observed. ${ }^{20}$ Figure 5 provides evidence that this leads to an increase in the DOS around $E_{F}$. This could also create additional unoccupied states that influence the relaxation process, hence accelerating the decay of the image potential states. Since image potential states are pinned to the vacuum level the work function increase for the oxygen covered surface increases the number of available final states for the decay process. This is also expected to shorten the image potential state lifetime. More dramatic change can take place for the $n=2$ IPS because on the O covered surface this state degenerates in energy with bulk states that leads to strong overlap with bulk states.

\section{CONCLUSIONS}

In summary we have investigated the population dynamics of IPS on the clean and oxygen covered Pt(111) surface. 
We have shown that experimental and theoretical data are in good agreement. After oxygen adsorption a $\operatorname{Pt}(111)-p(2$ $\times 2$ ) $-\mathrm{O}$ structure is formed leading to a quenching of the occupied surface resonance and a major rearrangement of the surface DOS. This is in turn mainly responsible for a faster depopulation of the IPS's due to an increased number of available final states.

\section{ACKNOWLEDGMENTS}

The authors gratefully acknowledge support from the TMR network Contract No. FMRX-CT-0178 and financial support from Max-Plank-Universität.
${ }^{1}$ N. Memmel and E. Bertel, Phys. Rev. Lett. 75, 485 (1995).

${ }^{2}$ P.M. Echenique and J.B. Pendry, J. Phys. C 11, 2065 (1978).

${ }^{3}$ N.V. Smith, Phys. Rev. B 32, 3549 (1985).

${ }^{4}$ D. Straub and F.J. Himpsel, Phys. Rev. Lett. 52, 1922 (1984).

${ }^{5}$ K. Giesen, F. Hage, F.J. Himpsel, H.J. Riess, and W. Steinmann, Phys. Rev. Lett. 55, 300 (1985).

${ }^{6}$ F. Passek, M. Donath, K. Ertl, and V. Dose, Phys. Rev. Lett. 75, 2746 (1995).

${ }^{7}$ W. Wallauer and Th. Fauster, Phys. Rev. B 54, 5086 (1996).

${ }^{8}$ M. Aeschlimann, M. Bauer, S. Pawlik, W. Weber, R. Burgermeister, D. Oberli, and H.-C. Siegmann, Phys. Rev. Lett. 79, 5158 (1997).

${ }^{9}$ R.W. Schoenlein, J.G. Fujimoto, G.L. Esley, and T.W. Capehart, Phys. Rev. Lett. 61, 2596 (1988).

${ }^{10}$ I.L. Shumay, U. Höfer, Ch. Reuß, U. Thomann, W. Wallauer, and Th. Fauster, Phys. Rev. B 58, 13974 (1998).

${ }^{11}$ T. Hertel, E. Knoesel, M. Wolf, and G. Ertl, Phys. Rev. Lett. 76, 535 (1996).

${ }^{12}$ E.V. Chulkov, I. Sarria, V.M. Silkin, J.M. Pitarke, and P.M. Echenique, Phys. Rev. Lett. 80, 4947 (1998).

${ }^{13}$ P.M. Echenique, J.M. Pitarke, E.V. Chulkov, and A. Rubio, Chem. Phys. 251, 1 (2000).

${ }^{14}$ J. Osma, I. Sarria, E.V. Chulkov, J.M. Pitarke, and P.M. Echenique, Phys. Rev. B 59, 10591 (1999).

${ }^{15}$ N. Fischer, S. Schuppler, Th. Fauster, and W. Steinmann, Phys. Rev. B 42, 9717 (1990).

${ }^{16}$ A. Schäfer, I.L. Shumay, M. Wiets, M. Weinelt, Th. Fauster, E.V. Chulkov, V.M. Silkin, and P.M. Echenique, Phys. Rev. B 61, 13 159 (2000).

${ }^{17}$ P. Roos, E. Bertel, and K.D. Rendulic, Chem. Phys. Lett. 232, 537 (1995).

${ }^{18}$ I. Kinoshita, T. Anazawa, and Y. Matsumoto, Chem. Phys. Lett. 259, 445 (1996).
${ }^{19}$ D.H. Parker, M.E. Bartram, and B.E. Koel, Surf. Sci. 217, 489 (1989).

${ }^{20}$ N. Materer, U. Starke, A. Barbieri, R. Döll, K. Heinz, M.A. Van Hove, and G.A. Somorjai, Surf. Sci. 325, 207 (1995).

${ }^{21}$ E. Wimmer, H. Krakauer, M. Weinert, and A.J. Freeman, Phys. Rev. B 24, 864 (1981); M. Weinert, E. Wimmer, and A.J. Freeman, ibid. 26, 4571 (1982).

${ }^{22}$ J.P. Perdew et al., Phys. Rev. B 46, 6671 (1992).

${ }^{23}$ Chun Li, A.J. Freeman, H.J.F. Jansen, and C.L. Fu, Phys. Rev. B 42, 5433 (1990).

${ }^{24}$ R. Drube, V. Dose, and A. Goldmann, Surf. Sci. 197, 317 (1988).

${ }^{25}$ E.V. Chulkov, V.M. Silkin, and P.M. Echenique, Surf. Sci. 437, 330 (1999), , 391, L1217 (1997).

${ }^{26}$ L. Hedin and S. Lundqvist, Solid State Phys. 23, 1 (1969).

${ }^{27}$ V.M. Silkin, E.V. Chulkov, and P.M. Echenique (unpublished).

${ }^{28}$ D. Straub and F.J. Himpsel, Phys. Rev. B 33, 2256 (1986).

${ }^{29}$ V.P. Chebotayev, High Resolution Laser Spectroscopy (Springer Verlag, Berlin, 1976).

${ }^{30} \mathrm{R}$. Loudon, The Quantum Theorie of Light (Oxford University Press, New York, 1983).

${ }^{31}$ J.C. Diels and W. Rudolph, Ultrashort Laser Pulse Phenomena (Academic Press, San Diego, 1996).

${ }^{32}$ A. Nebel and R. Beigang, Opt. Lett. 16, 1729 (1991).

${ }^{33}$ Y.I. Kang, Y.H. Cha, and C.H. Nam, Jpn. J. Appl. Phys. 38, 85 (1999).

${ }^{34}$ E.V. Chulkov, A. Liebsch, V.M. Silkin, and P.M. Echenique (unpublished).

${ }^{35}$ S. Schuppler, N. Fischer, Th. Fauster, and W. Steinmann, Appl. Phys. A: Solids Surf. 51, 322 (1990).

${ }^{36}$ J.D. McNeill, R.L. Lingle Jr., N.-H. Ge, C.M. Wong, R.E. Jordan, and C.B. Harris, Phys. Rev. Lett. 79, 4645 (1997).

${ }^{37}$ M. Wolf, E. Knoesel, and T. Hertel, Phys. Rev. B 54, R5295 (1996). 